\title{
Experimental issues and interpretation of the necking phenomena in the dynamic characterization via Hopkinson bar
}

\author{
Giuseppe Mirone ${ }^{\mathrm{a}}$ and Domenico Corallo \\ University of Catania, Dept. of Industrial Engineering, Viale A. Doria 6, 95125 Catania, Italy
}

\begin{abstract}
The usual procedures for processing experimental data from Hopkinson bars (HB) are based on modeling hypotheses which, in some cases, may lead to considerable approximations. The effects of the specimen geometry and of the necking are analysed, both experimentally and by FE analyses, for assessing how much the conventional values of strain rate, strain and stress, based on strain gauges readings along the HB, are affected by approximations in different testing conditions. Also, from the way the necking affects the load/area ratio, useful considerations arise about the hardening response of metal alloys under high strain rates. The sensitivity of stress-strain curves to the time-translations of strain waves along the bars is assessed by comparing the above curves to others where the strain, the strain rate and the current cross section come from speed camera image analyses. This clearly shows that the effective strain rate in the most strained areas of the specimens is many times higher than the nominal value, and also allows to explore how much the engineering curve is poorly representative of the material response, due to strain localization in the post-necking phase. The necking-affected length compared to the total gauge length is also found to significantly influence the strain response of the specimens, in a way that can easily mislead the experimenters in the derivation of the material ductility. An empirical relationship, already found to be valid in the post-necking quasi-static regime for transforming the true stress (load / current area) into an estimation of the cross section-averaged equivalent stress, is also found to apply at high strain rates if only the appropriate considerations are done on the way the equivalent stress is considered.
\end{abstract}

\section{Introduction}

The tensile tests performed at high strain rates by way of the Split Hopkinson Tension Bar (SHTB) are subjected to the same necking-induced stress-strain uniformity issues typical of the static tension tests, together with other possible inaccuracy causes, more directly inherent the strain-rate-dependent nature of the SHTB tests.

The reflected wave $\varepsilon \mathrm{r}$ obtained from strain gauge readings on the input bar only allows to derive the gross elongation rate of the entire specimen, including the contribution of fillets and shoulders always present in tensile specimens. Then the standard SHTB formulas are approximate due to the stress, the strain and the strain rate non-uniformities along the specimen length, up to very large extents depending on the geometry of the specimens.

Verleysen et al. [1,2] and Sato et al. [3] addressed this topic by providing a quantitative estimation of how the elongation rate and the engineering deformation, based on classical SHTB strain gauge readings on the bars, differ from the effective strain of the gage length of the specimen, based on image-analysis measurements.

Sato et al. [3] also evidenced the necking-induced progressive departure of the local true strain from the engineering strain, as it was also shown in Mirone [4] in terms of strain rate. When the strain measurements in SHTB experiments are only based on strain gauge readings, without speed camera image analysis, also the length of the specimen may greatly affect the calculation of

\footnotetext{
${ }^{a}$ Corresponding author: gmirone@dii.unict.it
}

the engineering strain, as also Rusinek et al. [5], Osovski et al. [6] and Rodriguez-Martinez et al. [7] pointed out. For this reason in [6] is proposes a "structural strain" for taking into account the strain localization induced by the necking.

Also the non-uniformity of stress distributions and its deviation from uniaxiality are directly related to the specimen shape, length and to the necking-induced perturbations, but the strain-rate related aspects of necking are somehow less investigated and still deserve further attention. In [4], starting from FE analyses of SHTB tests validated by literature experiments [17], was shown that the necking-affected experimental true stress, divided by the flow stress, obeyed to the same material-independent $M L R$ function suitable for the static case [18-21], if only the flow stress corresponding to the nominal engineering strain rate was considered through the adopted material model and the known material constants.

\section{Specimen geometry, necking and engineering vs. true curve approximation}

The most classical equations used in SHTB experiments for the stress / strain / strain rate characterization of materials are based on the elastic strain waves measured on the input bar (incident $\varepsilon_{i}$, reflected $\varepsilon_{r}$ ) and the output bar $\left(\operatorname{transmitted} \varepsilon_{t}\right)$ :

$$
\dot{\varepsilon}_{E n g}(t)=2 \cdot \frac{c_{0}}{L_{0}} \cdot \varepsilon_{r}(t)
$$

This is an Open Access article distributed under the terms of the Creative Commons Attribution License 4.0, which permits unrestricted use, distribution, and reproduction in any medium, provided the original work is properly cited. 


$$
\begin{gathered}
\varepsilon_{E n g}(t)=-2 \cdot \frac{c_{0}}{L_{0}} \cdot \int_{0}^{t} \varepsilon_{r}(t) \cdot d t \\
\sigma_{E n g}(t)=\frac{E_{b a r} \cdot A_{b a r}}{A_{0-S p}} \cdot \varepsilon_{t}(t)
\end{gathered}
$$

with $L_{0}$ reference gauge length of the smooth, unnotched specimen, $c_{0}$ the nominal sound speed in the bars $E, A_{b a r}$ and $A_{0-S p}$ the elastic modulus, the cross section of the bars and that of the undeformed smooth specimen, respectively. For a given SHTB system, the maximum achievable strain rate is inversely proportional to $L_{0}$, leading many researchers toward the adoption of specimens as short as possible, for increasing the achievable strain rate. This tendency is directly opposite to the need of long specimens arising from the hypothesis, intrinsic in Eqs. (1) and (2), that all the elongation of the specimen is concentrated within the gauge length $L_{0}$. In fact, $2 \cdot c_{0}$. $\varepsilon_{r}(t)$ in Eq. (1) expresses the time derivative of the total specimen length $L_{t o t}$, including the length of shoulders and fillets always present in tension samples. So, if the ratio $L_{0 /} A_{S p}$ is significant (slender, "long" specimens), then the elongation of the specimen shoulders is much smaller than that of the gauge length and the former can be neglected: only in this case Eq. (1) gives a good approximation of the engineering strain.

On the contrary, if the ratio $L_{0 /} A_{S p}$ is rather small (stub, "short" specimens), then the elongation of shoulders and fillets is comparable to that of the gage length and cannot be neglected, so Eq. (1) gives a poor approximation of the engineering strain.

This first requirement for long specimens in SHTB tests can only be overcome if the elongation of the gage length is recorded during a test, without including also the elongation of specimen fillets and shoulders. Given the speeds typical of SHTB, this can only be done by fast video acquisitions and image analysis, so that $\Delta L_{0 /} L_{0}$ and its time derivative are determined at known time intervals. Then, short specimens for increasing the achievable strain rate of SHTB tests can only be adopted if speed camera images are available, and in such a case Eqs. (1) and (2) should not be used.

Another aspect pushing toward the undesirable choice of "long" specimens is the necking-induced perturbation of the strain uniformity; the necking always affects a finite volume of the specimen roughly extending 2-3 times the undeformed diameter along the specimens axis. And, clearly, such necking-affected zone of the specimens deforms and elongates much more than the remaining segments of the gage length, if any. For whatever arbitrary given necking-affected segment $L_{N}$ of a specimen with its own local "true" strain $\log \left(A_{S p-0} / A_{N e c k}\right)$, the longer is the total gage length $L_{0}$, the smaller is the weight of the necking-elongated segment over the total gage length, the smaller is the engineering strain $\Delta L_{0 /} L_{0}$.

This is why shorter specimens apparently exhibit larger strains than longer ones; such difference is only apparent as local measurements of more pertinent true-strain $\varepsilon_{\text {True }}$, would show identical values at identical area reductions:

$$
\varepsilon_{\text {True }}(t)=\log \frac{A_{0-\text { Neck }}}{A_{\text {Neck }}} .
$$

Such second reason for adopting longer specimens is intrinsic to the necking phenomenon, it is also common to the static testing, and cannot be overcome in any way because, in principle, only infinitely long specimens would elongate with a negligible influence of the necking. The only possible countermeasure for this problem is that of adopting the true strain instead of the engineering strain; which, as before, can only be done by image analysis and fast video acquisition. The variability of the resisting cross section can be easily sorted out by fast video capture of the evolving neck section during experiments and successive image analysis:

$$
\sigma_{\text {True }}(t)=\frac{E_{b a r} \cdot A_{b a r}}{A_{S p}} \cdot \varepsilon_{t}(t) .
$$

The "true" stress $\sigma_{\text {True }}$ correctly describes the hardening only up to the inception of necking, after which the increasing stress triaxiality causes the true stress to considerably depart from the equivalent flow stress necessary for the material characterization.

The ratio $\sigma_{E q} / \sigma_{\text {True }}$ starts from 1 when the necking begins, and evolves by decreasing as the plastic strain flows, until fracture occurs. It has been shown that during the post-necking plastic straining up to failure, for the majority of structural metals such a ratio has always evolved in the range $0.75-1$ :

$$
\begin{aligned}
& \sigma_{E q}(t)<\sigma_{\text {True }}(t) \\
& 0.75<\frac{\sigma_{E q}(t)}{\sigma_{E q}(t)}<1 .
\end{aligned}
$$

The above ratio was found to be a material-independent function of the difference $\varepsilon_{\text {True }}-\varepsilon_{N}$, between the current true strain and the Considére strain (which, of course, is material-dependent), at which necking initiates.

The MLR polynomial in [18-21] approximates such a function within an error below 5\%, and then constitutes a tool for the necking correction of static true curves, which also resulted suitable for correcting dynamic true curves from SHTB as reported in $[4,19,20]$.

\section{Quantitative evaluation of length-induced and necking-induced approximations in classical SHTB tests}

Round bar specimens are machined with different lengths from 5 different metal alloys (Aluminium 2011, $A L$, Silicon Bronze $B R$, inox steel AISI 304, $X$, mild steel FE370, FE), according to the shape depicted in Fig. 1 and the nominal dimensions in Table 1.

The direct tension SHTB used consists of two Al 7075 bars with a $16 \mathrm{~mm}$ diameter, $3 \mathrm{~m}$ long the output bar, $4.5 \mathrm{~m}$ the input bar. A preload up to $100 \mathrm{kN}$ is imposed to the initial $1.5 \mathrm{~m}$ segment of the input bar and is released by fracturing a fragile sacrificial element which maintains closed a two-jaws gripping mechanism. A rise time of about $100 \mu \mathrm{s}$ is achieved. Signals acquisition is done by a $250 \mathrm{kHz} 8$ channels strain gauge station and a3 $\mathrm{MHz}$ digitalizing and sampling board.

Tests at different strain rates have been carried out either by imposing different incident waves on identical 


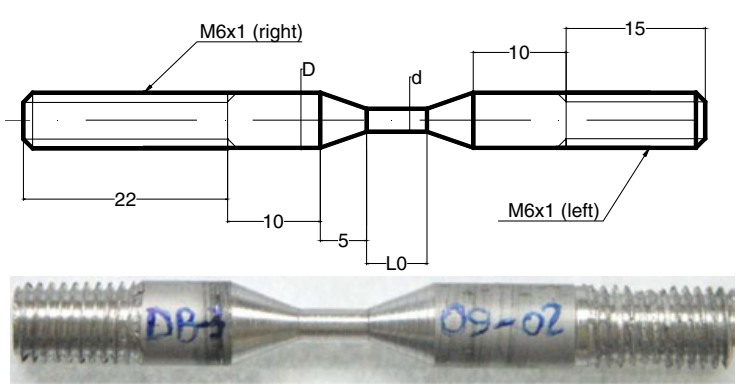

Figure 1. Specimens shape for multi-length, conventional SHTB tests.

Table 1. Specimens data for multi-length, conventional SHTB tests.

\begin{tabular}{|l|l|l|l|l|}
\hline Material & Spec. name & $d$ & $D$ & LO \\
\hline Alum. 2011 & AL-03-03 & 3 & 8 & 3 \\
\hline “ & AL-03-09 & 3 & 8 & 9 \\
\hline Bronze & ALs20-i & 2.5 & 6 & $2.5 / 6 / 10$ \\
\hline BR-03-03 & 3 & 8 & 3 \\
\hline AISI 304 & BR-03-09 & 3 & 8 & 9 \\
\hline X-03-03 & 3 & 8 & 3 \\
\hline FE370 & X-03-09 & 3 & 8 & 9 \\
\hline “ & FE-03-03 & 3 & 8 & 3 \\
\hline
\end{tabular}

specimens, or also by imposing the same incident wave to similar specimens of the same material, where only the gage length is varied. In this way the interacting effects of the elongation rate and of the specimen shape are investigated. This first series of standard tests is based on the classical SHTB theory, so only the engineering stress, strain and strain rates are evaluated according to Eqs. (1-3). Figures 2 and 3 show the results for the AL2011 alloy in terms of engineering stress-strain curves and of engineering strain rate vs. time, respectively. Short specimens $(L / d=1)$ only have a moderate dependency on the engineering strain rate; in fact, when $\varepsilon_{E n g}$ varies between 1000 and $7000 \mathrm{~s}^{-1}$, only a small variability within $20 \%$ in the ultimate stress and $15 \%$ in the failure strain occurs. A similar evidence is reported in Figs. 2b) and $3 \mathrm{~b})$ for the "long" specimens $(L / d=3)$. Instead the comparison between Figs. 2a) and $2 \mathrm{~b}$ ) suggests that the effect of $L / d$ is much more evident than that of the elongation rate; in fact, the static and dynamic failure strains of the short specimens in Fig. 2a) are almost identical each other, while in Fig. 2b), on the contrary, the dynamic failure strain is about 1.5 times greater than its static counterpart. In Figs. 2c) and 3c) three series of tests with the same incident wave (same preload of the input bar) and different L/d are compared; given that the effect of the engineering strain rate on specimens with the same shape is limited to within $20 \%$ as shown in Figs. 2a) and $2 b$ ), then the large differences between the three dynamic series in Fig. 2c) are only due to the different length/diameter ratio of the specimens.

It is clear that the material ductility at the local scale is not really increasing from about 0.2 to 0.7 when passing from long specimens to short ones, as suggested by Fig. 2c); such a wrong appearance, spoiling both static and

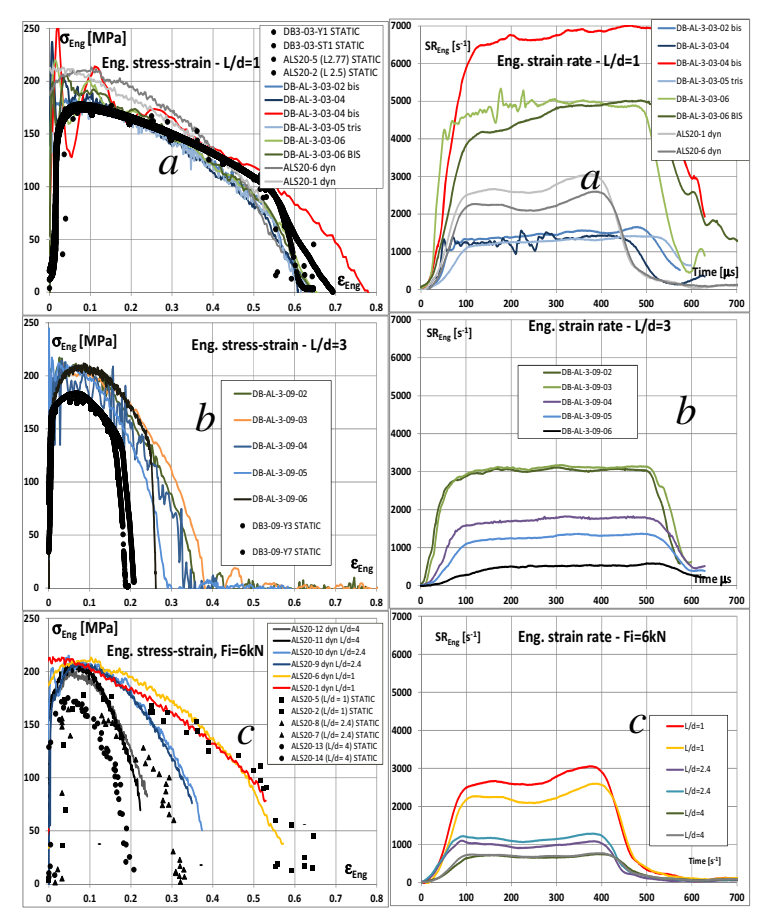

Figures 2-3. Engineering curves for AL.

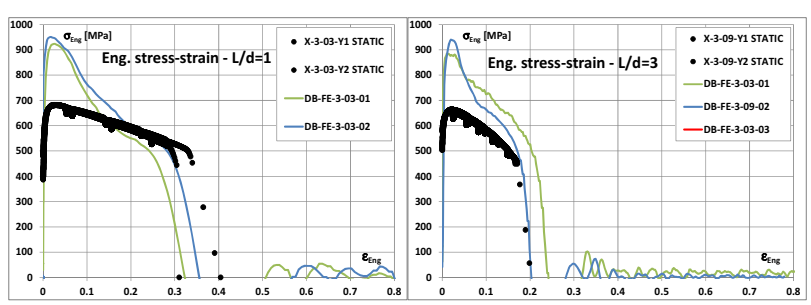

Figure 4. Engineering stress-strain curves for FE370.
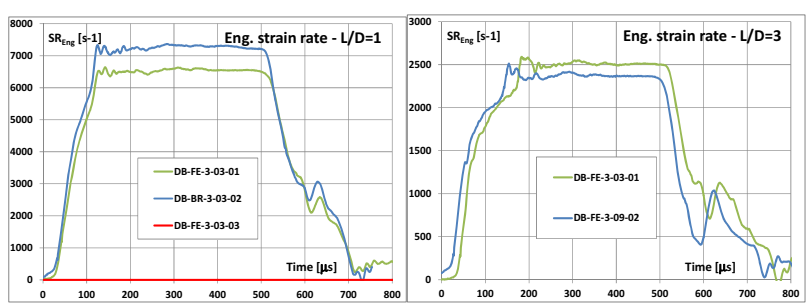

Figure 5. Engineering strain rate curves for FE370.

dynamic curves, is only a specimen-shape-dependent issue intrinsic in the engineering stress-strain curves.

Similar experiments are carried out with Bronze, AISI 304 and steel FE370, but only part of the respective results is reported below due to pages limit requirements.

The dependence of the hardening stress on the strain rate follows the usual predictable response as the former increases when the latter does, to different extents depending on the material sensitivity: the bronze hardening is almost strain-rate insensitive, while the $\mathrm{Al}$ alloy, the FE 37 and the AISI steels show strain-rate-promoted amplifications of the ultimate stress ranging from $12 \%$ to $28 \%$. The apparent increase of the engineering strain in too short specimens reflects how the elongation of the necked zone affects the elongation of the entire gage length, and its occurrence is evident for all materials at both static 
Table 2. Dynamic/static engineering ductility.

\begin{tabular}{|l|l|l|}
\hline & $\mathrm{L} / \mathrm{d}=1$ & $\mathrm{~L} / \mathrm{d}=3$ \\
\hline Al 2011 & 1 & 1.5 \\
\hline Bronze & 1 & 1.4 \\
\hline AISI304 & 0.65 & 0.85 \\
\hline FE370 & 0.95 & 1.05 \\
\hline
\end{tabular}

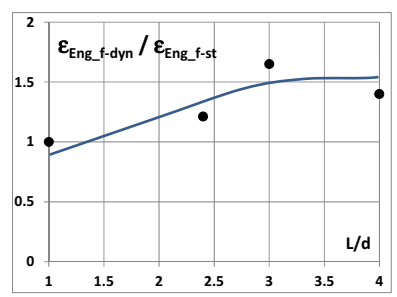

Figure 6. Specimen slenderness and strain increase.

and dynamics strain rates; instead, the magnitude of such apparent strain increase is related to the strain rate in a way that also depends on the material considered.

In fact, the ratio $\varepsilon_{E n g_{-} f_{-} D y n} / \varepsilon_{E n g_{-} f_{-} S t}$ between the average dynamic and static engineering strains at failure, reported in Table 2 against the L/D ratio for all materials, shows a similar trend for the non-ferrous alloys tested (in both $\mathrm{Al}$ and $\mathrm{Br}$ tests the failure strain has null increase for short specimens and a $50 \%$ increase for longer specimens), while it is rather different for the steels responses (ductility almost insensitive to the strain rate for the FE, regardless of the L/d ratio, and strain-rate-promoted ductility reduction for the AISI steel). The ratio $\varepsilon_{E n g_{-} f_{-} D y n} / \varepsilon_{E n g_{-} f_{-} S t}$ is plotted in Fig. 6 against the slenderness ratio $L / d$ for the Al alloy, the only one for which four different gauge lengths have been tested. More tests with many different $\mathrm{L} / \mathrm{d}$ ratios would be useful for a detailed identification of the above mechanism, but Fig. 6 already indicates that, for the alloy at hand, the effect of the specimen length on the engineering strain tends to become somehow stabilized at L/d of about 3; such a feature is not a real complete saturation and may either happen for other materials at different values of the slenderness ratio. Such experimental evidence is related to the considerations that the necked zone may largely affect very short specimens where the necking almost extends all over the entire gage length, while produces a less pronounced effect if specimens are longer and the necked zone only constitutes a partial or even a small segment of the entire gage length.

In Fig. 7 are reported two $\mathrm{Al}$ specimens with $\mathrm{L} / \mathrm{d}=$ 1 and 3 respectively, before tests and after failure. It is clearly visible that the length of the neck-affected zone is similar in both cases, but for shorter specimens it covers the entire gage length while for longer specimens it just covers about $50 \%$ of the total gage length. Then it follows that the necking in shorter specimens with $\mathrm{L} / \mathrm{d}=1$ affects the engineering strain to a maximum extent and, also, the interaction of the specimen shoulders with the gage length makes very likely that the uniaxiality of stress and the true stress-true strain curve are both spoiled well before of necking. Such short specimens behave more like notched bars than they do as smooth ones. The only way

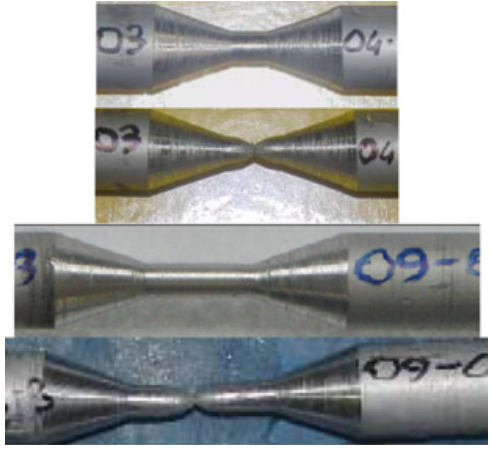

Figure 7. Neck-affected zone in short and long specimens.

for getting rid of these length-related issues is to rely on true stress and true strain measurements, provided that the gage lenght is long enough to prevent interaction of the specimen shoulders and fillets with the necked zone. Similar investigations conducted with the other alloys tested shown that a gage length two-three times longer than the diameter is essential for ensuring that a fraction of the gage length remains necking-free until failure, so that standard stress state of smooth unnotched specimen is achieved and virtually flawless true stress -true strain measurements can be performed.

The main results of the present section can then be summarized as follows:

- The gage length of specimens greatly affects the accuracy of engineering strain and strain rate measurements;

- The apparent increase in the engineering ductility of shorter specimens is only due to the amplified elongation of the necking zone, which in these cases either covers large amounts of the gage length or the entire gage length itself, so largely affecting the whole definition of engineering strain.

- When the necking covers the entire gage length it is very likely that the specimens behave as notched bars; then also the stress state at the local scale and the true stress-true strain data are affected by error.

- The length-induced spoiling of engineering strain measurements decreases and tends to saturate as the gage length increases, but such a saturation may be largely material-dependent and no real complete saturation is ensured at any length.

- On the contrary, the length-induced spoiling of true stress-true strain data only occurs when the entire gage length is affected by the necking; such a negative occurrence can be prevented by simply adopting L/d ratios greater of 2 or three, depending on the necking attitude of the given materials. It is understood that such a countermeasure poses a limitation on the maximum strain rate achievable on a given SHTB equipment for a given specimen diameter.

The analysis of the true stress-true strain characterization at high strain rates is the subject of investigation in the successive section. 


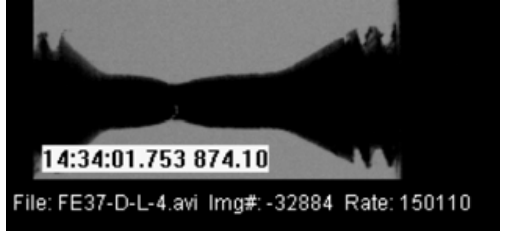

Figure 8. FEN specimen at incipient failure.

\section{Evidences of interaction between true stress, true strain and engineering strain rate}

A new series of tests has been carried out with an FE370 steel nominally identical to the previous one; however the material is obtained from a different supplier at a different time so that a slightly different response is found. The new FE370 steel is called FeN and the specimens have a ratio $\mathrm{L} / \mathrm{d}=2.5$. Two series of dynamic tests have been performed with the direct-tension SHTB apparatus, the "faster" series with an input bar preload of $50 \mathrm{kN}$ and a nominal $\dot{\varepsilon}_{E n g}=2500 \mathrm{~s}^{-1}$, the "slower" series with a bar preload of about $35 \mathrm{kN}$ generating a nominal $\dot{\varepsilon}_{\text {Eng }}=$ $1800 s^{-1}$. Fast image recordings were taken via a Phantom V7 speed camera at rates between $70 \mathrm{kfps}$ and $150 \mathrm{kfps}$, (Fig. 8), so that synchronization of frames with the load history from the output bar and successive image analysis allowed to calculate the engineering and the true stresses, strains and strain rates.

Huge difference between engineering curves and true curves are reported in Figs. 9 and 10 showing the results from the "camera-assisted" experiments. The most visible evidence conveyed by Figs. 9 and 10 is that the engineering curves hide and distort data over most of the material straining life; in fact, the apparent softening and the very low ductilities suggested by the engineering curves $\sigma_{E n g}\left(\varepsilon_{E n g}\right)$ are not real material features, but only inaccuracies intrinsic in the engineering approach, already known to afflict also the static elastic-plastic characterization. The failure strain form the engineering curves is close to $0.2-0.23$ while that from the true curves is about 5 times greater (0.9-1); also the stress is largely underestimated by the engineering curves, reporting stresses at failure of about $500 \mathrm{MPa}$ instead of the $800-1200 \mathrm{MPa}$ of the true stress range really exhibited by the material at incipient failure. Finally, the engineering strain rate is found to provide a huge underestimation of the true-strain rate, of a factor between 6 and 7; such an underestimation may radically change the outcome of a dynamic material characterization.

These considerations make clear why the knowledge of the true stress-true strain curves is an essential prerequisite for a reasonable material characterization, at both static and dynamic strain rates.

A commonly accepted hypothesis for the dynamic material characterization is that of assuming that the effects of strain and strain rate on the equivalent stress are uncoupled each other, in general:

$$
\sigma_{E q}\left(\varepsilon_{E q}, \dot{\varepsilon}_{E q}\right)=\sigma_{E q \_}\left(\varepsilon_{E q}\right) \cdot R\left(\dot{\varepsilon}_{E q}\right)
$$

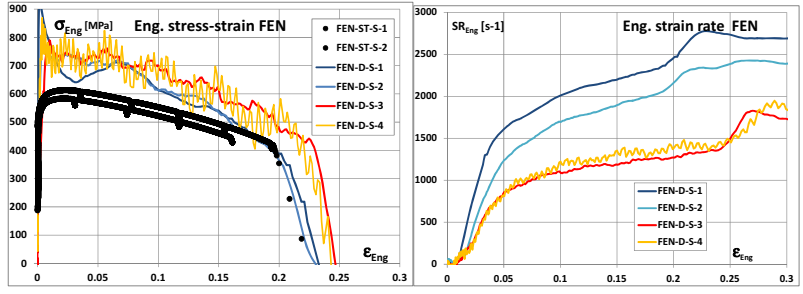

Figure 9. Engineering curves @ different strain rates.

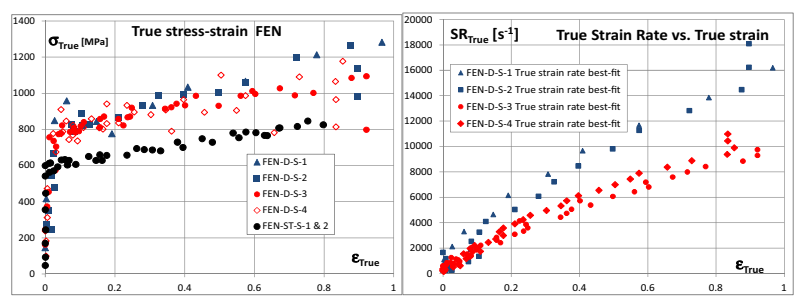

Figure 10. True curves@ different strain rates.

where $\sigma_{E q_{-} S}\left(\varepsilon_{E q}\right)$ is the static material curve and $R\left(\dot{\varepsilon}_{E q}\right)$ is the strain-rate-promoted amplification of the static hardening. Apart from the temperature effect which is not included here, widely known material models like those by Johnson-Cook or by Cowper-Symonds, are special cases of Eq. (1). Then the dynamic characterization consists of determining both right-hand terms of Eq. (7). The experimental measurement of quasi-static $\sigma_{E q_{S}}$ and $\varepsilon_{E q}$ is only possible from smooth tensile specimens before the necking initiates, when $\sigma_{E q}=\sigma_{\text {True }}$ and $\varepsilon_{E q}=\varepsilon_{\text {True }}$, respectively. After the necking starts $\varepsilon_{E q}$ remains quite close to $\varepsilon_{\text {True }}$, but $\sigma_{E q}$ progressively departs from $\sigma_{\text {True }}$.

The FEN steel exhibits early necking $\left(\varepsilon_{N} \sim 0.02 \div\right.$ 0.04 ) and rather long post-necking range (true strain at failure $\varepsilon_{\text {True }_{F}} \sim 0.9 \div 1$ at all the strain rates tested), so the departure between $\sigma_{E q}$ and $\sigma_{T r u e}$ is expected to be considerable. It was demonstrated in $[18,19]$ that the true stress and the equivalent stress at quasi-static rates are connected by the material-independent function $M L R$, according to Eq. (8):

$$
\sigma_{E q \_S}\left(\varepsilon_{E q}\right)=\sigma_{\text {True } \_}\left(\varepsilon_{E q}\right) \cdot \operatorname{MLR}\left(\varepsilon_{E q}-\varepsilon_{N}\right) .
$$

The ratio of dynamic true stress to static true stress has been plotted against the true strain rate for all the tests, as visible in the solid-line curves of Fig. 11a. Most of the literature, together with the material models mentioned before, assume that the stress amplification, expressed here by the ratio $R$, is a material characteristic function which, then, should not change for whatever strain-strain rate history occurring to the same metal; instead the experimental solid-line curves in Fig. 19a show two rather different trends of the ratio $R$ for the two series of tests, with a scatter of about $5 \%$ around the mean value. The reason of this difference cannot be explained at the moment, and it is not even clear whether or not it is physically sounding; however it is in contrast to the hypothesis expressed by Eq. (1), and suggests that different functions $R$ may exist or, which is more acceptable, that the function $R$ should also depend on $\varepsilon_{E q}$, so that the effects of the strain and of the strain rate is not uncoupled anymore. 


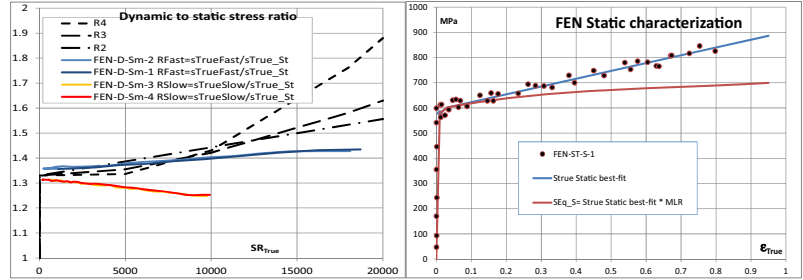

Figure 11. Static and dynamic stress-strain data.

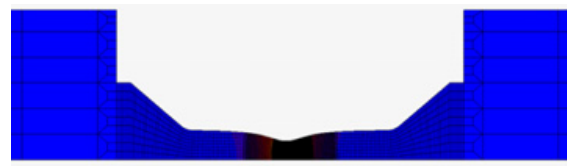

Figure 12. Deformed model of the SHTB specimen.

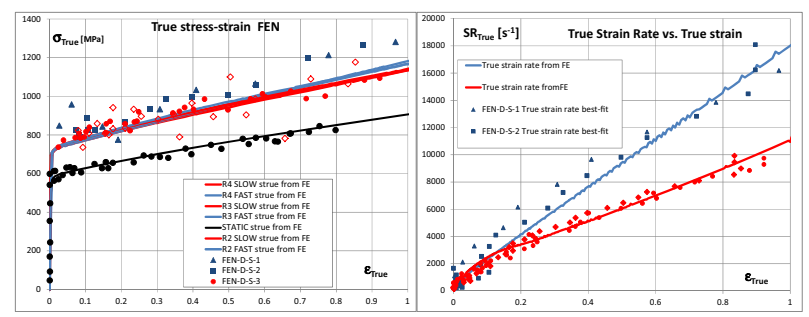

Figure 13. True stress, true strain and true strain rate from finite elements and from experiments.

Further detailed investigation on this subject is certainly deserved but lies beyond the timing of this work.

Then the dynamic characterization of the FEN alloy is attempted here by an inverse calibration procedure based on (1); the function $R$, adjusted iteratively, is joined to the Static flow curve from Eq. (8) until two FE analyses, driven by the same material parameters, are able to correctly simulate both the "slow" and the "fast" experiments. The three final choices of the $R$ ratio and the static flow curve are plotted as the black dashed curves in Fig. 11a and the lower curve of Fig. 11b, respectively.

The finite element model is made of 15900 axysimmetric quad-4 full integration elements for the input / output bars and the specimen. The material is described by Eq. (1) via the fortran user subroutine Yiel available within the MSC-MARC finite elements code. The output produced by the FE runs driven by the above material data is compared to the experimental data in Fig. 13.

While the true strain vs. true strain rate behavior is modeled correctly by finite elements for both dynamic tests, the simulated "fast" true curve from forces and displacements readings at the proper mesh nodes is rather underestimated. Only the simulated true curves for the static and the "slow" dynamic tests are satisfactory; and a very low sensitivity to the choice of $R$ is also found.

\section{Conclusions}

An experimental campaign based on static and dynamic SHTB tests has been carried out for investigating various aspects of the stress-strain characterization of metals at strain rates ranging from quasistatic to $20000 \mathrm{~s}-1$.
A first series of conventional SHTB tests has shown that the initial gage length of the specimen can significantly affect the test if the ratio $\mathrm{L} / \mathrm{d}$ is lower than about 2.5. These tests also highlighted the uncertainties intrinsic in the engineering definition of the stress-strain curves.

A second series of SHTB tests, including the acquisition of speed-camera images, allowed to determine the true stress-true strain curves and to calculate the truestrain rate at the neck section. The experimental true stresstrue strain curves fully highlighted the approximations and the lack of information conveyed by the engineering stressstrain data: for the FEN alloy, the standard strain-gaugebased SHTB procedures led to stress, strain and strain rate values about $2.5,5$ and 7 times smaller than their real values, respectively. The true strain rate vs. true strain curves obtained from finite elements are accurate, but the predicted true stress was accurate only for static and for slow dynamic tests; a visible true stress underestimation resulted from the simulation of fast dynamic tests.

\section{References}

[1] Verleysen P., Degrieck J., Int. J. Impact Engg, 2004. 30: $239-253$

[2] Sato K., Yu Q., Hiramoto J., Urabe T., Yoshitake A., Int. J Impact Engg 2015; 75: 11-26

[3] Mirone G. The dynamic effect of necking in Hopkinson bar tension tests. Mech. Mater. 2013. 58 84-96.

[4] A. Rusinek, R. Zaera, J.R. Klepaczko, R. Cheriguene, Acta Materialia 2005; 53: 5387-5400

[5] S. Osovski, D. Rittel, J.A. Rodríguez-Martínez, R. Zaera, Mechanics of Materials. 2013; 62 : 1-13

[6] J.A. Rodrígue z-Martínez, D. Rittel, R. Zaera, S. Osovski. 2013 ; 54:206-216

[7] Yang L.M., Shim V.P., Int J Impact Engg. 2005; 31:129

[8] K. Nilsson, Int. J Impact Engg. 2004; 30: 1353-1367

[9] Besnard G., Hild F., Lagrange J. M., Martinuzzi P., Roux S., International J Impact Engg. 2012; 49: 1353-1367

[10] R. Zaera, J.A. Rodríguez-Martínez, G. Vadillo, J. Fernández-Sáez, International Journal of Mechanics and Physics of Solids. 2014; 64: 316-337.

[11] Guan Zhiping. Materials and Design. 2014; 56: 209 218

[12] Z. Xue, A. Vaziri, J.W. Hutchinson. Journal of the Mechanics and Physics of Solids. 2008; 56: 93-113

[13] V. Tarigopula, O.S. Hopperstad, M. Langseth, A.H. Clausen, F. Hild, Int. J. Solids Struct. 45 (2008) 601619

[14] D. Anderson, S. Winkler, A. Bardelcik, M.J. Worswick, Materials and Design, 2014; 60:198-207

[15] C. Roth, Dirk Mohr, Int. J Plasticity. 2014; 56: 19-44

[16] G. Mirone, Int. J Sol. Struct., 2004; 41: 3545-3564

[17] G. Mirone, D. Corallo, Engg Fract. Mech., 2013; 102:118-145

[18] G. Mirone, Mech. Mat., 2008; 40: 685-694

[19] G. Mirone, Int. J. Damage Mech, 2004; 13: 241-261

[20] B. Erice, F. Gálvez, D.A. Cendón, V. SánchezGálvez, Engg Fract. Mech., 2012; 79: 1-17 